\title{
Asymptotic Plateau problem for prescribed mean curvature hypersurfaces
}

\section{Casteras, Jean-Baptiste}

2020-04

Casteras , J-B , Holopainen , I \& Ripoll , J B 2020 , ' Asymptotic Plateau problem for prescribed mean curvature hypersurfaces ' , Proceedings of the American Mathematical Society, vol. 148 , no. 4 , pp. 1731-1743 . https://doi.org/10.1090/proc/14829

http://hdl.handle.net/10138/312449

https://doi.org/10.1090/proc/14829

cc_by_nc_nd

acceptedVersion

Downloaded from Helda, University of Helsinki institutional repository.

This is an electronic reprint of the original article.

This reprint may differ from the original in pagination and typographic detail.

Please cite the original version. 


\title{
ASYMPTOTIC PLATEAU PROBLEM FOR PRESCRIBED MEAN CURVATURE HYPERSURFACES
}

\author{
JEAN-BAPTISTE CASTERAS, ILKKA HOLOPAINEN, AND JAIME B. RIPOLL
}

\begin{abstract}
We prove the existence of solutions to the asymptotic Plateau problem for hypersurfaces of prescribed mean curvature in Cartan-Hadamard manifolds $N$. More precisely, given a suitable subset $L$ of the asymptotic boundary of $N$ and a suitable function $H$ on $N$, we are able to construct a set of locally finite perimeter whose boundary has generalized mean curvature $H$ provided that $N$ satisfies the so-called strict convexity condition and that its sectional curvatures are bounded from above by a negative constant. We also obtain a multiplicity result in low dimensions.
\end{abstract}

\section{INTRODUCTION}

In this note we consider an asymptotic Plateau problem for hypersurfaces of prescribed generalized mean curvature and asymptotic behaviour at infinity on Cartan-Hadamard manifolds that have strictly negative curvature upper bound $-\alpha^{2}$ and satisfy the so-called strict convexity condition. We call such hypersurfaces as $H$-mean curvature hypersurfaces, where $H: N \rightarrow\left[-H_{0}, H_{0}\right]$ is a given continuous function, with $0 \leq H_{0}<(\operatorname{dim} N-1) \alpha$. Recall that a Cartan-Hadamard manifold $N$ is a complete, connected, and simply connected Riemannian $n$-manifold, $n \geq 2$, of non-positive sectional curvature. It can be compactified by adding a sphere at infinity $\partial_{\infty} N$ and equipping the union $N \cup \partial_{\infty} N$ with the cone topology; see 12 for the details. We say that $N$ satisfies the strict convexity condition ( $S C$ condition) if, given $x \in \partial_{\infty} N$ and a relatively open subset $W \subset \partial_{\infty} N$ containing $x$, there exists a $C^{2}$ open subset $\Omega \subset N$ such that $x \in \operatorname{int} \partial_{\infty} \Omega \subset W$ and $N \backslash \Omega$ is convex. Here int $\partial_{\infty} \Omega$ denotes the interior of $\partial_{\infty} \Omega$ in $\partial_{\infty} N$. For later purposes we also denote by bd the boundary relative to $\partial_{\infty} N$. The SC condition was introduced by Ripoll and Telichevesky (26]) in the context of an asymptotic Dirichlet problem for the minimal graph equation.

The $H$-mean curvature hypersurfaces are natural generalizations of minimal hypersurfaces. Anderson ([3]) solved the asymptotic Plateau problem in the hyperbolic space $\mathbb{H}^{n}$ for absolutely area minimizing varieties of arbitrary dimension and codimension. More precisely, he showed that, for any embedded closed submanifold $\Gamma^{p-1} \hookrightarrow \partial_{\infty} \mathbb{H}^{n}$ in the sphere at infinity, there exists a complete, absolutely area minimizing locally integral $p$-current $\Sigma \subset \mathbb{H}^{n}$ asymptotic to $\Gamma^{p-1}$ at infinity. By interior regularity results from geometric measure theory (see [13, 24]), $\Sigma$ is smoothly embedded in $\mathbb{H}^{n}$ provided that $p=n-1 \leq 6$. Using similar methods based on mass minimizing currents, Bangert and Lang extended Anderson's results to Riemannian

2000 Mathematics Subject Classification. 53A10, 53C42, 49Q05, 49Q20.

Key words and phrases. Hadamard manifolds, asymptotic Plateau problem.

J.-B.C. supported by the FNRS project MIS F.4508.14; I.H. supported by the Väisälä Fund and the Magnus Ehrnrooth foundation; J.R. supported by the CNPq (Brazil) project 302955/2011-9. 
manifolds that are diffeomorphic and bi-Lipschitz equivalent to a Cartan-Hadamard manifold that has pinched sectional curvature $-b^{2} \leq K \leq-1$ ([5]) or that is Gromov hyperbolic and has bounded geometry $(20])$. In these results the topology of the minimal submanifold is not controlled. We refer to [4, 11, 22, 28, for existence results of complete minimal hypersurfaces with prescribed topology.

Our research is inspired by the papers of Alencar and Rosenberg [1] and of Tonegawa 30] who studied the asymptotic Plateau problem for constant mean curvature hypersurfaces in the hyperbolic space. Tonegawa also investigated the regularity up to the boundary. For existence results of constant mean curvature hypersurfaces in $\mathbb{H}^{n}$ that can be represented as graphs we refer to $14,25,27$. We refer to [8, 10] for a survey and recent developments on the $H$-Plateau problem in the hyperbolic space. In this paper we obtain the hypersurfaces $M$ of generalized mean curvature $H$ as reduced boundaries of sets of locally finite perimeter $Q$ in $N$ that locally minimizes a family of functionals

$$
\int_{Q \cap C} H(x) d m(x)+\int_{\partial^{*} Q \cap C} d \mathcal{H}^{n-1}
$$

for all compact sets $C \subset N$.

In [6] we searched geometric conditions on $N$ that would imply the $\mathrm{SC}$ condition. We were able to verify the SC condition on manifolds whose curvature lower bound could go to $-\infty$ and upper bound to 0 simultaneously at certain rates, or on some manifolds whose sectional curvatures go to $-\infty$ faster than any prescribed rate. For instance, the SC condition holds on $N$ if

or

$$
-\frac{e^{2 k r(x)}}{r(x)^{2+2 \varepsilon}} \leq K_{N}(x) \leq-k^{2}
$$

$$
-c e^{(2-\varepsilon) r(x)} e^{e^{r(x) / e^{3}}} \leq K_{N}(x) \leq-\phi e^{2 r(x)}
$$

for all $x \in N$ such that $r(x)=d(x, o) \geq R^{*}, R^{*}$ large enough, and where $k>0, \varepsilon>$ $0, \phi>1 / 4$, and $c>0$ are constants. We refer to [6. Theorem 1.3] for more precise conditions. The SC condition was also applied in [6] to solve asymptotic Dirichlet and Plateau problems on Cartan-Hadamard manifolds.

Our main results read as follows.

Theorem 1.1. Let $N^{n}$ be a Cartan-Hadamard manifold satisfying the SC condition. Suppose also that sectional curvatures of $N$ have a negative upper bound

$$
K_{N} \leq-\alpha^{2}<0, \quad \alpha>0 .
$$

Let $L \subset \partial_{\infty} N$ be a relatively closed subset such that $L=\operatorname{bd} U=\operatorname{bd} U^{\prime}$ for some disjoint relatively open subsets $U, U^{\prime} \subset \partial_{\infty} N$ with $U \cup U^{\prime}=\partial_{\infty} N \backslash L$. Suppose that $H: N \rightarrow\left[-H_{0}, H_{0}\right]$ is a continuous function where $0 \leq H_{0}<(n-1) \alpha$. Then there exists an open set $Q \subset N$ of locally finite perimeter whose boundary $M$ has (generalized) mean curvature $H$ towards $N \backslash Q, \partial_{\infty} Q \subset U \cup L$, and $\partial_{\infty} M=L$.

Following [1, we also obtain a multiplicity result:

Theorem 1.2. Let $N^{n}$ and $L \subset \partial_{\infty} N$ be as above. If $\left.\left.H \in\right] 0, H_{0}\right]$ is a constant, there exist two disjoint open sets $Q_{j} \subset N, j=1,2$, of locally finite perimeter whose boundaries $M_{j}$ have constant (generalized) mean curvature $H$ and $\partial_{\infty} M_{j}=L$. Moreover, if $n \leq 7$, then $M_{1}$ and $M_{2}$ are disjoint. 
It seems that some kind of convexity condition is necessary to solve the asymptotic Plateau problem. Indeed, Kloeckner and Mazzeo [18, Corollary 5.2] proved, in the case $N^{3}=\mathbb{H}^{2} \times \mathbb{R}$, which clearly does not satisfy the SC condition, that the asymptotic Plateau problem for the geodesic compactification (the one described above), is solvable only for very specific families of curves $L$. On a related topic, Coskunuzer 9 also obtained non-existence results in the case $N^{3}=\mathbb{H}^{2} \times \mathbb{R}$ but with the product compactification i.e. $\partial_{\infty} N=\left(\partial_{\infty} \mathbb{H}^{2} \times \mathbb{R}\right) \cup\left(\partial_{\infty} \mathbb{H}^{2} \times \partial_{\infty} \mathbb{R}\right) \times\left(\mathbb{H}^{2} \times \partial_{\infty} \mathbb{R}\right)$. A similar result has been obtained in [17] when $N$ is the homogenuous space $\mathbb{E}(-1, \tau)$. Notice that in all the previous examples, the sectional curvatures are null in some directions. The asymptotic Plateau problem for minimal hypersurfaces might be solvable on manifolds satisfying, instead of the SC condition, a strict mean convexity condition, where the convexity of sets $N \backslash \Omega$ is replaced by mean convexity. We also believe that it should be possible to allow the sectional curvatures of $N$ to go to 0 not too fast (as in our results concerning the asymptotic Plateau problem for minimal submanifolds [6]). But currently, we do not know how to prove Lemma 3.1 in such settings; see in particular (3.1).

The plan of this note is the following: in Section 2 we introduce the variational problem we are going to consider and prove the first variation formula for smooth hypersurfaces. Using the first variation formula we then obtain a maximum principle. Then, in Section 3 we apply the SC condition and the curvature upper bound to prove a "trapping" lemma (Lemma 3.1). Then we consider the asymptotic $H$ Plateau problem and prove our main results.

Acknowledgement. We wish to thank the referee for suggestions, which led to improvements in the presentation.

\section{Prescribed (Generalized) mean curvature}

Let $N^{n}$ be an $n$-dimensional Cartan-Hadamard manifold. Suppose that $B=$ $B(o, R)$ is a geodesic (open) ball of radius $R$ centered at $o \in N$ and that $\Sigma \subset \partial B$ is a relatively open set, with $\mathcal{H}^{n-2}$-rectifiable boundary $\Gamma=\partial \Sigma$ (relative to $\partial B$ ). Consider open sets $Q \subset N$ of finite perimeter and with (topological) boundary

$$
\partial Q=M \cup \Gamma \cup \Sigma,
$$

where $M \subset N$ is an $\mathcal{H}^{n-1}$-rectifiable topological, not necessarily connected, $(n-1)$ manifold.

Given a continuous function $H: N \rightarrow \mathbb{R}$ we consider the following (Massari) functional $\mathcal{F}$,

$$
\mathcal{F}(M)=\int_{Q} H(x) d m(x)+\int_{M} d \mathcal{H}^{n-1},
$$

where $M$ and $Q$ are as above. We call such $M$ admissible for the functional $\mathcal{F}$ with boundary data $\Gamma$.

We call $H$ the prescribed mean curvature function. The terminology is justified by the fact that a stationary $M$ of the functional (2.1) has mean curvature $H(x)$ pointing outwards $Q$ at every point $x \in M$ where $M$ is smooth; see e.g. [23] and [21, 17.3]. We refer to [21, Theorem 17.5 and Prop. 17.8] for first variation formulae of perimeter and potential energy. For our purposes, the first variation formula (2.2) for a smooth hypersurface $M$ is sufficient. For the convenience of reader's we sketch its proof. To this end, suppose that $M$ is smooth and admissible for (2.1) 
with boundary data $\Gamma$. Let $X$ be a smooth vector field such that $X=0$ on $\Gamma$ and let $\Phi$ be the flow of $X$. For sufficiently small $|t|$ we denote $M_{t}=\{\Phi(x, t): x \in M\}$ and by $Q_{t}$ the set bounded by $M_{t} \cup \Gamma \cup \Sigma$. To obtain the first variation formula for smooth $M$ we compute

$$
\begin{aligned}
\delta \mathcal{F}(M)[X] & :=\frac{d}{d t} \mathcal{F}\left(M_{t}\right)_{\mid t=0} \\
& =\frac{d}{d t}\left(\int_{Q_{t}} H(x) d m(x)+\int_{M_{t}} d \mathcal{H}^{n-1}\right)_{\mid t=0} \\
& =\frac{d}{d t}\left(\int_{Q_{t}} H(x) d m(x)\right)_{\mid t=0}+\int_{M} \operatorname{div}_{M} X d \mathcal{H}^{n-1} \\
& =\frac{d}{d t}\left(\int_{Q_{t}} H(x) d m(x)\right)_{\mid t=0}-\int_{M}\langle X, \vec{H}\rangle d \mathcal{H}^{n-1},
\end{aligned}
$$

where $\vec{H}$ is the mean curvature vector field of $M$. To compute the first term on the right-hand side we apply the co-area formula. For that purpose we define in spt $X$ and for sufficiently small $|t|$ a smooth function $f$ by setting $f(x)=t$ for $x \in M_{t}$. Thus $f=0$ and $\nabla f=X^{\perp} /\left|X^{\perp}\right|^{2}$ on $M=M_{0}$. Hence

$$
\begin{aligned}
& \lim _{t \rightarrow 0} \frac{1}{t}\left(\int_{Q_{t}} H(x) d m(x)-\int_{Q} H(x) d m(x)\right) \\
= & \lim _{t \rightarrow 0} \frac{1}{t}\left(\int_{Q_{t} \backslash Q}|\nabla f(x)||\nabla f(x)|^{-1} H(x) d m(x)\right. \\
& \left.\quad-\int_{Q \backslash Q_{t}}|\nabla f(x)||\nabla f(x)|^{-1} H(x) d m(x)\right) \\
= & \int_{M}\left\langle X_{x}, \nu_{x}\right\rangle H(x) d \mathcal{H}^{n-1}(x),
\end{aligned}
$$

where $\nu_{x}$ is the unit normal to $M$ at $x$ pointing outwards $Q$. To verify the last step above, we may assume $t \rightarrow 0^{+}$. Then the set $Q_{t} \backslash Q$, whenever nonempty for small $t>0$, corresponds those $x \in M$ where $X_{x}$ points outwards $Q$, that is $\left\langle X_{x}, \nu_{x}\right\rangle>0$. The co-area formula now implies

$$
\begin{aligned}
& \lim _{t \rightarrow 0+} \frac{1}{t} \int_{Q_{t} \backslash Q}|\nabla f(x)||\nabla f(x)|^{-1} H(x) d m(x) \\
= & \lim _{t \rightarrow 0+} \frac{1}{t} \int_{0}^{t}\left(\int_{M_{s} \cap\left(Q_{t} \backslash Q\right)}|\nabla f(x)|^{-1} H(x) d \mathcal{H}^{n-1}(x)\right) d s \\
= & \int_{M \cap\{\langle X, \nu\rangle>0\}}\left\langle X_{x}, \nu_{x}\right\rangle H(x) d \mathcal{H}^{n-1}(x) .
\end{aligned}
$$

Similarly,

$$
\begin{aligned}
\lim _{t \rightarrow 0+} \frac{1}{t} \int_{Q \backslash Q_{t}} & |\nabla f(x)||\nabla f(x)|^{-1} H(x) d m(x) \\
& =-\int_{M \cap\{\langle X, \nu\rangle<0\}}\left\langle X_{x}, \nu_{x}\right\rangle H(x) d \mathcal{H}^{n-1}(x) .
\end{aligned}
$$


Thus we obtain the first variation formula

$$
\delta \mathcal{F}(M)[X]=\int_{M}\left\langle X_{x}, H(x) \nu_{x}-\vec{H}_{x}\right\rangle d \mathcal{H}^{n-1}(x) .
$$

Assuming that a smooth hypersurface $M$ is stationary, i.e.

$$
\delta \mathcal{F}(M)[X]=0
$$

for all $X$ as above, we conclude that $M$ has prescribed mean curvature $H(x)$ pointing outwards $Q$.

Fix $o \in N$ and denote by $H(x, r)$ the scalar (inwards pointing) mean curvature of the geodesic sphere $S(o, r)=\partial B(o, r)$ at $x \in S(o, r)$. Hence $H(x, r)=\Delta r(x)$, where $r(x)=d(x, o)$, the Riemannian distance between $o$ and $x$. Suppose that the prescribed mean curvature function $H$ satisfies

$$
|H(x)|<H(x, r)=\Delta r(x)
$$

for all $x \in S(o, r)$ and $r>0$.

We have the following maximum principle which is the counterpart of 15 , Lemma $5]$, where the proof is given in dimension three in a slightly different setting.

Lemma 2.1. Let $M \subset N$ be admissible for $\mathcal{F}$ with boundary data $\Gamma \subset \partial B, B=$ $B(o, R)$, such that $M \backslash \bar{B} \neq \emptyset$. Then there exists $M^{\prime} \subset \bar{B}$ admissible for $\mathcal{F}$ with boundary data $\Gamma$ such that $\mathcal{F}\left(M^{\prime}\right)<\mathcal{F}(M)$. In particular, if $M_{0}$ is a minimizer of the functional (2.1) with boundary data $\Gamma$, then $M_{0} \subset \bar{B}$.

Proof. Let $Q$ be the open set corresponding the set $M$ and let $U=Q \backslash \bar{B}$. Note that $U$ is a nonempty open set, and therefore it has positive volume. By [2, Theorem $3.42]$ there are open sets $Q_{i}$ with smooth boundaries such that $Q_{i}$ converges to $Q$ in measure and $\mathcal{H}^{n-1}\left(\partial Q_{i}\right)=P\left(Q_{i}\right) \rightarrow P(Q)$ as $i \rightarrow \infty$. Here $P(Q)$ stands for the perimeter of $Q$. Hence we may suppose that there exists a sequence of smooth $(n-1)$-manifolds $M_{i}$ admissible for $\mathcal{F}$ with boundary data $\Gamma$ such that $M_{i} \backslash \bar{B} \neq$ $\emptyset, M_{i} \subset B(o, R+s)$ for some $s>0$, and $\mathcal{F}\left(M_{i}\right) \rightarrow \mathcal{F}(M)$. Therefore it is enough to show that for each $M_{i}$ there exists $M_{i}^{\prime} \subset \bar{B}$ admissible for $\mathcal{F}$ with boundary data $\Gamma$ such that $\mathcal{F}\left(M_{i}^{\prime}\right) \leq \mathcal{F}\left(M_{i}\right)-c$ for some positive constant $c$ independent of $i$. More precisely, we prove that

$$
\mathcal{F}\left(M_{i}^{\prime}\right) \leq \mathcal{F}\left(M_{i}\right)-\frac{1}{2} \int_{U_{i}}((1-\varepsilon) \Delta r(x)-|H(x)|) d m(x),
$$

where $U_{i}=Q_{i} \backslash \bar{B}$ and $\varepsilon>0$ is such that $|H(x)|<(1-\varepsilon) \Delta r(x)$ for all $x \in$ $\bar{B}(o, R+s)$. Since $Q_{i}$ converges to $Q$ in measure, we obtain an admissible $M^{\prime} \subset \bar{B}$ as the Hausdorff limit of $M_{i}^{\prime}$ 's, with

$$
\mathcal{F}\left(M^{\prime}\right) \leq \mathcal{F}(M)-\frac{1}{4} \int_{U}((1-\varepsilon) \Delta r(x)-|H(x)|) d m(x)<\mathcal{F}(M) .
$$

The integral in (2.5) is positive since $U$ has positive volume and $(1-\varepsilon) \Delta r-|H|$ is positive in $U$.

To prove (2.4) we fix $M_{i}$ and let $x_{1}$ be a point of $M_{i}$ at the maximal distance from $o$. Then the mean curvature vector $\vec{H}_{x_{1}}$ of $M_{i}$ at $x_{1}$ is parallel to $-\nabla r\left(x_{1}\right)$ and $\left|\vec{H}_{x_{1}}\right| \geq \Delta r\left(x_{1}\right)$. Let $r_{1}$ be the infimum of all $r \geq R$ such that

$$
\left\langle\vec{H}_{x}, \nabla r(x)\right\rangle<0 \quad \text { and } \quad\left|\vec{H}_{x}\right| \geq(1-\varepsilon) \Delta r(x)>|H(x)|
$$


for all $x \in M_{i}$ with $r(x) \geq r$. We notice that $r_{1}<r\left(x_{1}\right)$. Next we define a complete smooth vector field $X_{1}$ on $N$ by setting $X_{1}(x)=-\rho_{1}(r(x)) \nabla r(x)$, where $\rho_{1}:[0, \infty) \rightarrow[0, \infty)$ is a smooth function such that $\rho_{1}(s)>0$ if and only if $s>r_{1}$. Let $\Phi_{1}: \mathbb{R} \times N \rightarrow N$ be the flow of $X_{1}$ and denote

$$
M_{i}^{t}=\left\{\Phi_{1}(t, x): x \in M_{i}\right\}
$$

for $t>0$. Furthermore, let

$$
\begin{aligned}
U_{i, 1} & =\left\{\Phi_{1}(t, x): t>0, x \in M_{i} \backslash \bar{B}\left(o, r_{1}\right)\right\} \\
& =\left\{\exp _{o} t v: r_{1} /|v|<t<1, v \in \exp _{o}^{-1}\left(M_{i} \backslash \bar{B}\left(o, r_{1}\right)\right)\right\}
\end{aligned}
$$

be the open set whose boundary is contained in $\left(M_{i} \backslash \bar{B}\left(o, r_{1}\right)\right) \cup \partial B\left(o, r_{1}\right)$. Notice that $U_{i, 1} \subset U_{i}$ by (2.6), in fact, $U_{i, 1}=U_{i} \backslash \bar{B}\left(o, r_{1}\right)$. Finally, let $\widetilde{M}_{i, 1}$ be the Hausdorff limit of $M_{i}^{t}$ as $t \rightarrow \infty$. Thus

$$
\widetilde{M}_{i, 1}=\left(M_{i} \cap \bar{B}\left(o, r_{1}\right)\right) \cup\left\{\exp _{o}\left(r_{1} v /|v|\right): v \in \exp _{o}^{-1}\left(M_{i} \backslash \bar{B}\left(o, r_{1}\right)\right)\right\}
$$

the union of $M_{i} \cap \bar{B}\left(o, r_{1}\right)$ and the radial projection of $M_{i} \backslash \bar{B}\left(o, r_{1}\right)$ into $\partial B\left(o, r_{1}\right)$. Notice that $M_{i} \cap B\left(o, r_{1}\right)=\widetilde{M}_{i, 1}$. Using (2.6) and the co-area formula we obtain

$$
\begin{aligned}
\mathcal{F}\left(\widetilde{M}_{i, 1}\right) & =\mathcal{F}\left(M_{i}\right)+\int_{0}^{\infty} \delta \mathcal{F}\left(M_{i}^{t}\right)\left[X_{1}\right] d t \\
& =\mathcal{F}\left(M_{i}\right)-\int_{0}^{\infty}\left(\int_{M_{i, 1}^{t}}\left\langle X_{1}(x), \vec{H}_{x}-H(x) \nu_{x}\right\rangle d \mathcal{H}^{n-1}(x)\right) d t \\
& \leq \mathcal{F}\left(M_{i}\right)-\int_{U_{i, 1}}((1-\varepsilon) \Delta r(x)-|H(x)|) d m(x) .
\end{aligned}
$$

To verify the inequality in (2.7) we define a smooth function $f$ on $U_{i, 1}$ by setting $f=t$ on $M_{i, 1}^{t} \cap U_{i, 1}$ and apply the co-area formula with the function $f$. Now $\nabla f=X_{1}^{\perp} /\left|X_{1}^{\perp}\right|^{2}$ on $M_{i, 1}^{t} \cap U_{i, 1}$, where $X_{1}^{\perp}$ is the orthogonal component of $X_{1}$ with respect to $M_{i, 1}^{t}$. Thus

$$
|\nabla f(x)|^{-1}=\left|X_{1}^{\perp}(x)\right|=\left\langle X_{1}(x), \vec{H}_{x} /\left|\vec{H}_{x}\right|\right\rangle
$$

for $x \in M_{i, 1}^{t}$, where $\vec{H}$ is the mean curvature vector field of $M_{i, 1}^{t}$. Since $\left|\vec{H}_{x}\right|>$ $|H(x)|$ and $\vec{H}_{x}$ is parallel to $-\nu_{x}$, the vector $\vec{H}_{x}$ is parallel to $\vec{H}_{x}-H(x) \nu_{x}$, and therefore

$$
\left\langle X_{1}(x), \vec{H}_{x}-H(x) \nu_{x}\right\rangle=-\rho_{1}(x)\left\langle\nabla r(x), \vec{H}_{x}-H(x) \nu_{x}\right\rangle>0
$$

in $U_{i, 1}$. More precisely,

$$
\begin{aligned}
\left\langle X_{1}(x), \vec{H}_{x}-H(x) \nu_{x}\right\rangle & \geq\left(\left|\vec{H}_{x}\right|-|H(x)|\right)\left\langle X_{1}, \vec{H}_{x} /\left|\vec{H}_{x}\right|\right\rangle \\
& \geq((1-\varepsilon) \Delta r(x)-|H(x)|)|\nabla f(x)|^{-1}
\end{aligned}
$$


in $U_{i, 1}$. We obtain

$$
\begin{aligned}
\int_{U_{i, 1}} & ((1-\varepsilon) \Delta r(x)-|H(x)|) d m(x) \\
& =\int_{U_{i, 1}}((1-\varepsilon) \Delta r(x)-|H(x)|)|\nabla f(x)|^{-1}|\nabla f(x)| d m(x) x \\
& =\int_{0}^{\infty}\left(\int_{M_{i, 1}^{t}}((1-\varepsilon) \Delta r(x)-|H(x)|)|\nabla f(x)|^{-1} d \mathcal{H}^{n-1}(x)\right) d t \\
& \leq \int_{0}^{\infty}\left(\int_{M_{i, 1}^{t}}\left\langle X_{1}(x), \vec{H}_{x}-H(x) \nu_{x}\right\rangle d \mathcal{H}^{n-1}(x)\right) d t
\end{aligned}
$$

and the estimate (2.7) follows.

If $r_{1}=R$, we obtain (2.4) since $\widetilde{M}_{i, 1} \subset \bar{B}$ and $U_{i, 1}=U_{i}$ in that case. If $r_{1}>R$ we continue by smoothing out $\widetilde{M}_{i, 1}$ in a neighborhood of $M_{i} \cap \partial B\left(o, r_{1}\right)$ to obtain a smooth $M_{i, 1} \subset U_{i} \cap \bar{B}\left(o, r_{1}\right)$ admissible for $\mathcal{F}$ with boundary data $\Gamma$. We denote by $Q_{i, 1}$ the open set bounded by $M_{i, 1} \cap \Gamma \cap \Sigma$. We also write $\widetilde{Q}_{i, 1}=Q_{i} \cap B\left(o, r_{1}\right)$ and $A_{i, 1}=\widetilde{Q}_{i, 1} \backslash Q_{i, 1}$. The smoothing can be done such that the volume of $A_{i, 1}$ and $\mathcal{H}^{n-1}\left(M_{i, 1} \backslash \widetilde{M}_{i, 1}\right)$ are as small as we wish, in particular, so that

$$
\begin{aligned}
\mathcal{F}\left(M_{i, 1}\right)-\mathcal{F}\left(\widetilde{M}_{i, 1}\right) & \leq-\int_{A_{i, 1}} H(x) d m(x)+\mathcal{H}^{n-1}\left(M_{i, 1} \backslash \widetilde{M}_{i, 1}\right) \\
& <\frac{1}{4} \int_{U_{i}}((1-\varepsilon) \Delta r(x)-|H(x)|) d m(x)
\end{aligned}
$$

We repeat the argument above by choosing a point $x_{2} \subset M_{i, 1}$ at the maximal distance from $o$ and letting $r_{2}$ be the infimum of $r \geq R$ such that (2.6) holds for all $x \in M_{i, 1}$, with $r(x) \geq r$. Then $r_{2}<r_{1}$ by the smoothing process. We obtain an admissible $\widetilde{M}_{i, 2} \subset \bar{B}\left(o, r_{2}\right)$ with

$$
\mathcal{F}\left(\widetilde{M}_{i, 2}\right) \leq \mathcal{F}\left(M_{i, 1}\right)-\int_{U_{i, 2}}((1-\varepsilon) \Delta r(x)-|H(x)|) d m(x),
$$

where

$$
U_{i, 2}=\left\{\exp _{o} t v: r_{1} /|v|<t<1, v \in \exp _{o}^{-1}\left(M_{i, 1} \backslash \bar{B}\left(o, r_{2}\right)\right)\right\} \subset U_{i} .
$$

If $r_{2}=R$, we are done. Otherwise we smooth out $\widetilde{M}_{i, 2} \subset \bar{B}\left(o, r_{2}\right)$ to obtain an admissible smooth $M_{i, 2} \subset \bar{B}\left(o, r_{2}\right)$ with

$$
\mathcal{F}\left(M_{i, 2}\right)-\mathcal{F}\left(\widetilde{M}_{i, 2}\right)<\frac{1}{4^{2}} \int_{U_{i}}((1-\varepsilon) \Delta r(x)-|H(x)|) d m(x) .
$$

By continuing this way we get a strictly decreasing (possible finite) sequence $r_{j} \geq R$ and admissible $\widetilde{M}_{i, j}, M_{i, j} \subset \bar{B}\left(o, r_{j}\right)$, where $M_{i, j}$ is smooth, such that

$$
\begin{aligned}
\mathcal{F}\left(M_{i, j}\right)<\mathcal{F}\left(M_{i, j-1}\right) & -\int_{U_{i, j}}((1-\varepsilon) \Delta r(x)-|H(x)|) d m(x) \\
& +\frac{1}{4^{j}} \int_{U_{i}}((1-\varepsilon) \Delta r(x)-|H(x)|) d m(x) .
\end{aligned}
$$


At each step $r_{j}$ is the infimum of $r \geq R$ such that (2.6) holds for all $x \in M_{i, j-1}$, with $r(x) \geq r$. Hence

$$
\begin{aligned}
\mathcal{F}\left(M_{i, j}\right)<\mathcal{F}\left(M_{i}\right) & -\int_{\cup_{k=1}^{j} U_{i, k}}((1-\varepsilon) \Delta r(x)-|H(x)|) d m(x) \\
& +\sum_{k=1}^{j}\left(\frac{1}{4}\right)^{k} \int_{U_{i}}((1-\varepsilon) \Delta r(x)-|H(x)|) d m(x) \\
<\mathcal{F}\left(M_{i}\right)- & \int_{\bigcup_{k=1}^{j} U_{i, k}}((1-\varepsilon) \Delta r(x)-|H(x)|) d m(x) \\
& +\frac{1}{3} \int_{U_{i}}((1-\varepsilon) \Delta r(x)-|H(x)|) d m(x) .
\end{aligned}
$$

If $r_{k}=R$ for some $k$, we are done. Similarly, if $r_{\infty}:=\lim _{j} r_{j}=R$, the Hausdorff limit $M_{i}^{\prime}$ of $M_{i, j}$ 's is admissible, $M_{i}^{\prime} \subset \bar{B}$, and satisfies

$$
\begin{aligned}
\mathcal{F}\left(M_{i}^{\prime}\right)<\mathcal{F}\left(M_{i}\right) & -\int_{\cup_{j} U_{i, j}}((1-\varepsilon) \Delta r(x)-|H(x)|) d m(x) \\
& +\frac{1}{3} \int_{U_{i}}((1-\varepsilon) \Delta r(x)-|H(x)|) d m(x) .
\end{aligned}
$$

On the other hand, if $r_{\infty}:=\inf r_{j}>R$, the Hausdorff limit $M_{i}^{\prime} \subset \bar{B}\left(o, r_{\infty}\right), M_{i}^{\prime} \cap$ $\partial B\left(o, r_{\infty}\right) \neq \emptyset$, and $(2.8)$ holds. Repeating the process once more gives a contradiction with the definition of $r_{\infty}$. Moreover, the smoothing processes can be done so that the set $U_{i} \backslash\left(\cup_{j} U_{i, j}\right)$ has as small volume as we wish; see [29]. Finally, since the sets $U_{i}$ converge to $U$ in measure, we obtain $M^{\prime} \subset \bar{B}$ admissible for $\mathcal{F}$ with boundary data $\Gamma$ such that

$$
\mathcal{F}\left(M^{\prime}\right) \leq \mathcal{F}(M)-\frac{1}{4} \int_{U}(\Delta r(x)-|H(x)|) d m(x) .
$$

Remark 2.2. (1) In fact, the constant $1 / 4$ in (2.5) can be replaced by 1 . Indeed, instead of the constant ratio $1 / 4$ in geometric series we may use a constant $q>0$ as small as we wish and perform the smoothing processes so that the lost of volume is as small as we like. Then $1 / 4$ in (2.5) can be replaced by any constant $c<1$, hence also by 1 .

(2) Consider open sets $Q \subset N$ of finite perimeter with topological boundary of the form $\partial Q=M \cup \Gamma \cup \Sigma$, where $\Sigma$ is a relatively compact $\mathcal{H}^{n-1}$ rectifiable topological $(n-1)$-manifold whose relative boundary $\Gamma=\partial \Sigma$ is $\mathcal{H}^{n-2}$-rectifiable. We define the (Massari) functional $\mathcal{F}$ as in (2.1) also in this setting. Suppose that $\Gamma \subset \bar{B}(o, R)$ for some $R>0$. The proof of Lemma 2.1 then gives that any minimizer of $\mathcal{F}$ with boundary data $\Gamma$ must stay inside $\bar{B}(o, R)$.

(3) The existence of minimizers is proven below right before Lemma 3.1 .

\section{Asymptotic $H$-Plateau problem}

Throughout this section we assume that $N$ satisfies the $\mathrm{SC}$ condition and that the sectional curvatures of $N$ are bounded from above by a negative constant $-\alpha^{2}$. 
Suppose, furthermore, that

$$
|H(x)| \leq H_{0}<(n-1) \alpha .
$$

Then there exists $r_{0}$ such that $(n-1) \alpha \tanh \left(\alpha r_{0}\right)>H_{0}$. If $A \subset N$ is an open convex set, we denote by $A_{0}$ the (open) $r_{0}$-neighborhood of $A$ and we write $A_{0}^{+}=N \backslash \overline{A_{0}}$.

Let $U$ and $U^{\prime}$ be disjoint relatively open sets in $\partial_{\infty} N$ such that $U \cup U^{\prime}=\partial_{\infty} N \backslash L$, where $L=\operatorname{bd} U=\operatorname{bd} U^{\prime}$. Furthermore, let $O$ be the (open) tubular neighborhood of Cone $_{o} L$ with fixed positive radius $\delta$ and let $\widetilde{O}$ be the tubular neighborhood of Cone $_{o} U$ with the same radius $\delta$. Note that $O \subset \widetilde{O}$. As in [6, Lemma 4.1] we take, for each $x \in \partial_{\infty} N \backslash L$, a relatively open set $W_{x} \subset \partial_{\infty} N \backslash L$ such that $x \in W_{x}$ and then apply the SC condition and the Hessian comparison to obtain a $C^{2}$ smooth open subset $\Omega_{x}$ of $N$ such that $x \in \operatorname{int} \partial_{\infty} \Omega_{x} \subset W_{x}, N \backslash \Omega_{x}$ is convex, and $\Omega_{x} \cap O=\emptyset$ for every $x \in \partial_{\infty} N \backslash L$.

We define

$$
\begin{aligned}
& \Lambda=\bigcap_{x \in \partial_{\infty} N \backslash L}\left(N \backslash \Omega_{x}\right), \\
& \widetilde{\Lambda}=\bigcap_{x \in U^{\prime}}\left(N \backslash \Omega_{x}\right) .
\end{aligned}
$$

Then $O \subset \Lambda, \widetilde{O} \subset \widetilde{\Lambda}, \Lambda$ and $\widetilde{\Lambda}$ are closed in $N$, Int $\Lambda$ and $\operatorname{Int} \widetilde{\Lambda}$ are open convex subsets of $N$ such that $\partial_{\infty} \Lambda=L$ and $\partial_{\infty} \widetilde{\Lambda}=L \cup U$.

For a fixed $R>0$ we approximate $\partial B(o, R) \cap \widetilde{O}$ by a relatively open subset $\Sigma \subset$ $\partial B(o, R)$ so that its boundary $\Gamma:=\partial \Sigma$ is an $\mathcal{H}^{n-2}$-rectifiable subset of $\partial B(o, R) \cap O$. We consider $[\Sigma]$ as a rectifiable $(n-1)$-current and $[\Gamma]=\partial[\Sigma]$ as a rectifiable $(n-2)$-current. Let $M_{j}, j \in \mathbb{N}$, be a minimizing sequence for the functional (2.1) with boundary data $\Gamma$. Then there exists a subsequence, still denoted by $M_{j}$, and an $\mathcal{H}^{n-1}$-rectifiable set $M$ such that $\left[M_{j}\right] \rightarrow[M]$ and $\partial[M]=[\Gamma]$ as currents. It follows that $M$ is a minimizer for (2.1) with boundary data $\Gamma$, hence $M \subset \bar{B}(o, R)$ by Lemma 2.1. Next we prove that $M \subset \bar{B}(o, R) \cap \Lambda_{0}$, where $\Lambda_{0}$ is the $r_{0}$-neighborhood of $\Lambda$, cf. [7, Theorem 1.1].

Lemma 3.1. Let $\Gamma \subset \partial B(o, R) \cap O$ be the boundary of $\Sigma \subset \partial B(o, R) \cap \widetilde{O}$ as above. Then there exists a minimizer $M$ of (2.1) with the boundary data $\Gamma$ such that $M \subset \bar{B}(o, R) \cap \bar{\Lambda}_{0}$.

Proof. The existence of a minimizer $M$ was obtained above. By Lemma 2.1, it suffices to prove that $M \subset \Lambda_{0}$. Hence it is enough to show that, for all $x \in \partial_{\infty} N \backslash L$, $M \subset \overline{\left(N \backslash \Omega_{x}\right)_{0}}$, where $\left(N \backslash \Omega_{x}\right)_{0}$ is the $r_{0}$-neighborhood of the $\left(C^{2}\right.$-smooth $)$ convex set $N \backslash \Omega_{x}$. Let $d=\operatorname{dist}\left(\cdot, N \backslash \Omega_{x}\right)$ be the Riemannian distance to the convex set $N \backslash \Omega_{x}$. Then $d$ is $C^{2}$-smooth in $N \backslash \Omega_{x}$ and the Hessian comparison theorem [16] implies that

$$
\Delta d(z) \geq(n-1) \alpha \tanh (\alpha d(z)) \geq(n-1) \alpha \tanh \left(\alpha r_{0}\right)>H_{0} \geq|H(z)|
$$

for all $z \in\left(N \backslash \Omega_{x}\right)_{0}^{+}=N \backslash \overline{\left(N \backslash \Omega_{x}\right)_{0}}$. Assume on the contrary that $M \cap\left(N \backslash \Omega_{x}\right)_{0}^{+} \neq$ $\emptyset$. As in the proof Lemma 2.1 there is a sequence of smooth $(n-1)$-manifolds $M_{i}$ admissible for the functional $\mathcal{F}$ with boundary data $\Gamma$ such that $M_{i} \cap\left(N \backslash \Omega_{x}\right)_{0}^{+} \neq \emptyset$ and $\mathcal{F}\left(M_{i}\right) \rightarrow \mathcal{F}(M)$. Again, for each fixed $M_{i}$, if $x_{1} \in M_{i}$ is at maximal distance from $N \backslash \Omega_{x}$, the mean curvature vector $\vec{H}_{x_{1}}$ of $M_{i}$ is parallel to $-\nabla d\left(x_{1}\right)$ and $\left|\vec{H}_{x_{1}}\right| \geq \Delta d\left(x_{1}\right)>H\left(x_{1}\right)$. Hence $\left\langle\vec{H}_{x}, \nabla d(x)\right\rangle<0$ and $\left|\vec{H}_{x}\right|>|H(x)|$ for all 
$x \in M_{i}$ with $d(x) \geq d_{1}=d\left(x_{1}\right)-\varepsilon$. Next we define a smooth vector field on $N$ by setting $X_{x}=-\rho(d(x)) \nabla d(x)$, where $\rho:[0, \infty) \rightarrow[0, \infty)$ is a smooth non-decreasing function such that $\rho(t)>0$ if and only if $t>d_{1}$. Then $\left\langle X_{x}, H(x) \nu_{x}-\vec{H}_{x}\right\rangle<0$ for $d(x)>d_{1}$ and vanishes elsewhere. Applying the first variation formula to $M_{i}$ with the vector field $X$ we obtain

$$
\delta \mathcal{F}\left(M_{i}\right)[X]=\int_{M_{i}}\left\langle X_{x}, H(x) \nu_{x}-\vec{H}_{x}\right\rangle d \mathcal{H}^{n-1}(x)<0 .
$$

In particular, deforming $M_{i}$ in the direction of $X$ decreases the value of the functional and repeating a similar argument as in the proof of Lemma 2.1 we may conclude that the minimizer $M$ must stay inside $\left(N \backslash \Omega_{x}\right)_{0}$.

Now we are ready to prove our first main theorem. For convenience we repeat it from Introduction.

Theorem 3.2. Let $N^{n}$ be a Cartan-Hadamard manifold satisfying the $S C$ condition. Suppose also that sectional curvatures of $N$ have a negative upper bound

$$
K_{N} \leq-\alpha^{2}<0, \quad \alpha>0 .
$$

Let $L \subset \partial_{\infty} N$ be a relatively closed subset such that $L=\operatorname{bd} U=\operatorname{bd} U^{\prime}$ for some disjoint relatively open subsets $U, U^{\prime} \subset \partial_{\infty} N$ with $U \cup U^{\prime}=\partial_{\infty} N \backslash L$. Suppose that $H: N \rightarrow\left[-H_{0}, H_{0}\right]$ is a continuous function where $0 \leq H_{0}<(n-1) \alpha$. Then there exists an open set $Q \subset N$ of locally finite perimeter whose boundary $M$ has (generalized) mean curvature $H$ towards $N \backslash Q, \partial_{\infty} Q \subset U \cup L$, and $\partial_{\infty} M=L$.

Proof. The proof is an adaptation of a method of Lang [19]; see also [5] and [6]. We apply Lemma 3.1 in geodesic balls $B\left(o, R_{i}\right)$, where $R_{i} \rightarrow \infty$ is an increasing sequence. For each $i$ we approximate $\partial B\left(o, R_{i}\right) \cap \widetilde{O}$ by a relatively open subset $\Sigma_{i} \subset \partial B\left(o, R_{i}\right)$ so that $\Gamma_{i}:=\partial \Sigma_{i} \subset \partial B\left(o, R_{i}\right) \cap O$ is $\mathcal{H}^{n-2}$-rectifiable. Furthermore, let $M_{i} \subset \bar{B}\left(o, R_{i}\right) \cap \Lambda_{0}$ be a minimizer of (2.1) with the boundary data $\Gamma_{i}$ given by Lemma 3.1. Then $\bar{M}_{i} \cup \Sigma_{i}$ bounds an open set $Q_{i}$ of finite perimeter. Furthermore, total variations are locally uniformly bounded as

$$
\sup _{i}\left\|\partial\left[Q_{i}\right]\right\|(B(o, r)) \leq \mathcal{H}^{n-1}(\partial B(o, r))+H_{0} \operatorname{Vol}(B(o, r))
$$

the upper bound depending only on $r, n$, and the sectional curvature lower bound on $B(o, r)$. Hence there exists a subsequence $Q_{i_{j}}$ and a set $Q \subset N$ of locally finite perimeter with boundary $M=\partial Q$ such that $\left[Q_{i_{j}}\right] \rightarrow[Q]$ and $\left[M_{i_{j}}\right] \rightarrow[M]$ as currents and that $M$ is a minimizer for $\mathcal{F}$ in the sense that $\delta \mathcal{F}(M)[X]=0$ for all compactly supported smooth vector fields. Hence $M$ has (generalized) mean curvature $H$ pointing outwards $Q$. Furthermore, since $M \subset \Lambda_{0}$ and $\partial_{\infty} \Lambda_{0}=L$, we have $\partial_{\infty} M \subset L$. To prove that $L \subset \partial_{\infty} M$, take an arbitrary point $x \in L$ and let $V$ be a (cone) neighborhood of $x$. Then there exists a geodesic $\gamma: \mathbb{R} \rightarrow N$, a closed tubular neighborhood $K$ of $\gamma(\mathbb{R})$, with $K \subset V$, and points at infinity $\gamma(-\infty) \in U \cap V, \gamma(\infty) \in U^{\prime} \cap V$. Hence for all sufficiently large $i, K$ intersects both $\partial B\left(o, R_{i}\right) \cap$ Cone $_{o} U$ and $\partial B\left(o, R_{i}\right) \cap$ Cone $_{o} U^{\prime}$, and consequently $\left[\Gamma_{i}\right.$ ] can not be a boundary of a rectifiable $(n-1)$-current $T$ with $\operatorname{spt} T \subset \bar{B}\left(o, R_{i}\right) \backslash K$. In particular, $M_{i}$ intersects with $V$ for all sufficiently large $i$, and since $V$ is an arbitrary neighborhood of $x$, we conclude that $x \in \partial_{\infty} M$. Hence $L \subset \partial_{\infty} M$.

Remark 3.3. Notice that, by regularity theory, a minimizer $M$ of the functional (2.1) is a $C^{2}$-smooth $(n-1)$-dimensional manifold up to a closed singular set of 
Hausdorff dimension at most $n-8$; see e.g. 23, Teorema 5.1, 5.2]. In particular, in dimensions $n \leq 7$, a minimizer $\mathrm{M}$ is $C^{2}$-smooth.

The next result is a generalization of [1, Theorem 3].

Theorem 3.4. Let $N^{n}$ be a Cartan-Hadamard manifold satisfying the SC condition. Suppose also that sectional curvatures of $N$ have a negative upper bound

$$
K_{N} \leq-\alpha^{2}<0, \quad \alpha>0 .
$$

Let $L \subset \partial_{\infty} N$ be a relatively closed subset such that $L=\operatorname{bd} U=\operatorname{bd} U^{\prime}$ for some disjoint relatively open subsets $U, U^{\prime} \subset \partial_{\infty} N$ with $U \cup U^{\prime}=\partial_{\infty} N \backslash L$. If $\left.H \in\right] 0, H_{0}$ ] is a constant, there exist two disjoint open sets $Q_{j} \subset N, j=1,2$, of locally finite perimeter whose boundaries $M_{j}$ have constant (generalized) mean curvature $H$ and $\partial_{\infty} M_{j}=L$.

Proof. Let $Q_{1} \subset N$, with $\partial_{\infty} Q_{1} \subset U \cup L$, be an open set of locally finite perimeter obtained in (the proof of) Theorem 1.1. Thus its boundary $M_{1}$ has constant (generalized) mean curvature towards $N \backslash Q_{1}$. Repeating the construction with $\Sigma_{i}$ replaced by $\Sigma_{i}^{\prime}=\partial B\left(o, R_{i}\right) \backslash\left(\Sigma_{i} \cup \Gamma_{i}\right)$ gives another open set $Q_{2}$ of locally finite perimeter whose boundary $M_{2}$ has constant mean curvature $H$ towards $N \backslash Q_{2}$. Furthermore, $\partial_{\infty} Q_{2} \subset U^{\prime} \cup L$ and $\partial_{\infty} M_{j}=L$. In the special case $H \equiv 0$, Theorem 1.1 yields the existence of an open set $W \subset N$ of locally finite perimeter such that $S=\partial[W]$ is a (mass) minimizing locally rectifiable $(n-1)$-current (with integer multiplicity) and $\partial_{\infty}$ spt $S=L$; see also [6, Theorem 1.6]. Then $Q_{1} \subset W$ and $Q_{2} \subset N \backslash \bar{W}$ since otherwise it would be possible to strictly decrease the value of the functional $\mathcal{F}$ by replacing $M_{1} \cap(N \backslash \bar{W})$ with spt $S \cap Q_{1}$, and similarly for $Q_{2}, M_{2}$. Hence $Q_{1} \cap Q_{2}=\emptyset$.

Remark 3.5. Although $Q_{1} \cap Q_{2}=\emptyset$, the above reasoning does not imply that the boundaries $M_{1}$ and $M_{2}$ are (pointwise) disjoint. We believe that $M_{1} \cap M_{2}=\emptyset$ but, unfortunately, we are not able to prove it in full generality. However, using the regularity results from geometric measure theory it is possible to prove that the intersection $M_{1} \cap M_{2}$ is rather small and, in particular, an empty set in dimensions $n \leq 7$; see Remark 3.3 ,

Theorem 3.6. Let $Q_{i}$ and $M_{i}, i=1,2$, be given by Theorem 1.2. Denote by $S_{i}$ the closed singular sets of Hausdorff dimension at most $n-8$ such that $M_{i} \backslash S_{i}, i=1,2$, are $C^{2}$-smooth submanifolds. Then $\left(M_{1} \backslash S_{1}\right) \cap\left(M_{2} \backslash S_{2}\right)=\emptyset$. In particular, in dimensions $n \leq 7$, the sets $S_{i}$ are empty, and consequently $M_{1}$ and $M_{2}$ are disjoint.

Proof. Suppose on the contrary that $\left(M_{1} \backslash S_{1}\right) \cap\left(M_{2} \backslash S_{2}\right) \neq \emptyset$ and let $x \in\left(M_{1} \backslash\right.$ $\left.S_{1}\right) \cap\left(M_{2} \backslash S_{2}\right)$. Then $M_{i} \backslash S_{1}$ has constant mean curvature $H$ at $x$ towards $N \backslash Q_{i}$. Now both $M_{1}$ and $M_{2}$ are subsets of $N \backslash Q_{2}$ intersecting tangentially at $x$. Since $M_{1}$ has mean curvature $-H$ at $x$ towards $N \backslash Q_{2}$ and $M_{2}$ has mean curvature $H$ at $x$ towards $N \backslash Q_{2}$, we get a contradiction since $H>0$.

\section{REFERENCES}

[1] Alencar, H., And Rosenberg, H. Some remarks on the existence of hypersurfaces of constant mean curvature with a given boundary, or asymptotic boundary, in hyperbolic space. Bull. Sci. Math. 121, 1 (1997), 61-69.

[2] Ambrosio, L., Fusco, N., And Pallara, D. Functions of bounded variation and free discontinuity problems. Oxford Mathematical Monographs. The Clarendon Press, Oxford University Press, New York, 2000. 
[3] Anderson, M. T. Complete minimal varieties in hyperbolic space. Invent. Math. 69, 3 (1982), $477-494$.

[4] Anderson, M. T. Complete minimal hypersurfaces in hyperbolic n-manifolds. Comment. Math. Helv. 58, 2 (1983), 264-290.

[5] Bangert, V., And Lang, U. Trapping quasiminimizing submanifolds in spaces of negative curvature. Comment. Math. Helv. 71, 1 (1996), 122-143.

[6] Casteras, J.-B., Holopainen, I., and Ripoll, J. B. Convexity at infinity in CartanHadamard manifolds and applications to the asymptotic Dirichlet and Plateau problems. Math. Z. 290, 1-2 (2018), 221-250.

[7] Coskunuzer, B. Minimizing constant mean curvature hypersurfaces in hyperbolic space. Geom. Dedicata 118 (2006), 157-171.

[8] Coskunuzer, B. Asymptotic Plateau problem: a survey. In Proceedings of the Gökova Geometry-Topology Conference 2013 (2014), Gökova Geometry/Topology Conference (GGT), Gökova, pp. 120-146.

[9] Coskunuzer, B. Minimal surfaces with arbitrary topology in $\mathbb{H}^{2} \times \mathbb{R}$. Preprint arXiv:1404.0214v2 [math.DG] (2014).

[10] Coskunuzer, B. Embedded $H$-planes in hyperbolic 3-space. Trans. Amer. Math. Soc. 371, 2 (2019), 1253-1269.

[11] de Oliveira, G., And Soret, M. Complete minimal surfaces in hyperbolic space. Math. Ann. 311, 3 (1998), 397-419.

[12] Eberlein, P., And O'Neill, B. Visibility manifolds. Pacific J. Math. 46 (1973), 45-109.

[13] Federer, H. Geometric measure theory. Die Grundlehren der mathematischen Wissenschaften, Band 153. Springer-Verlag New York Inc., New York, 1969.

[14] Guan, B., And Spruck, J. Hypersurfaces of constant mean curvature in hyperbolic space with prescribed asymptotic boundary at infinity. Amer. J. Math. 122, 5 (2000), 1039-1060.

[15] Gulliver, II, R. D. The Plateau problem for surfaces of prescribed mean curvature in a Riemannian manifold. J. Differential Geometry 8 (1973), 317-330.

[16] Kasue, A. A Laplacian comparison theorem and function theoretic properties of a complete Riemannian manifold. Japan. J. Math. (N.S.) 8, 2 (1982), 309-341.

[17] Klaser, P., Menezes, A., and Ramos, A. On the asymptotic Plateau problem for area minimizing surfaces in $\mathbb{E}(-1, \tau)$. Preprint arXiv:1905.03191 [math.DG] (2019).

[18] Kloeckner, B. R., and Mazzeo, R. On the asymptotic behavior of minimal surfaces in $\mathbb{H}^{2} \times \mathbb{R}$. Indiana Univ. Math. J. 66, 2 (2017), 631-658.

[19] LANG, U. The existence of complete minimizing hypersurfaces in hyperbolic manifolds. Internat. J. Math. 6, 1 (1995), 45-58.

[20] LANG, U. The asymptotic Plateau problem in Gromov hyperbolic manifolds. Calc. Var. Partial Differential Equations 16, 1 (2003), 31-46.

[21] Maggi, F. Sets of finite perimeter and geometric variational problems, vol. 135 of Cambridge Studies in Advanced Mathematics. Cambridge University Press, Cambridge, 2012. An introduction to geometric measure theory.

[22] Martín, F., and White, B. Properly embedded, area-minimizing surfaces in hyperbolic 3-space. J. Differential Geom. 97, 3 (2014), 515-544.

[23] Massari, U. Esistenza e regolarità delle ipersuperfice di curvatura media assegnata in $R^{n}$. Arch. Rational Mech. Anal. 55 (1974), 357-382.

[24] Morgan, F. Geometric measure theory, fourth ed. Elsevier/Academic Press, Amsterdam, 2009. A beginner's guide.

[25] Nelli, B., AND SpRuck, J. On the existence and uniqueness of constant mean curvature hypersurfaces in hyperbolic space. In Geometric analysis and the calculus of variations. Int. Press, Cambridge, MA, 1996, pp. 253-266.

[26] Ripoll, J., And Telichevesky, M. Regularity at infinity of Hadamard manifolds with respect to some elliptic operators and applications to asymptotic Dirichlet problems. Trans. Amer. Math. Soc. 367, 3 (2015), 1523-1541.

[27] Ripoll, J., And Telichevesky, M. On the asymptotic Plateau problem for CMC hypersurfaces in hyperbolic space. Bull. Braz. Math. Soc. (N.S.) (2018).

[28] Ripoll, J., And Tomi, F. Complete minimal discs in Hadamard manifolds. Adv. Calc. Var. 10, 4 (2017), 315-330

[29] Schmid, T. Strict interior approximation of sets of finite perimeter and functions of bounded variation. Proc. Amer. Math. Soc. 143, 5 (2015), 2069-2084. 
[30] Tonegawa, Y. Existence and regularity of constant mean curvature hypersurfaces in hyperbolic space. Math. Z. 221, 4 (1996), 591-615.

Département de Mathématique, Université Libre de Bruxelles, CP 214, Boulevard du Triomphe, B-1050 Bruxelles, Belgium

E-mail address: jeanbaptiste.casteras@gmail.com

Department of Mathematics and Statistics, P.O. Box 68, 00014 University of Helsinki, FinLAND

E-mail address: ilkka.holopainen@helsinki.fi

Ufrgs, Instituto de Matemática, Av. Bento Goncalves 9500, 91540-000 Porto AlegreRS, BRASIL

E-mail address: jaime.ripoll@ufrgs.br 